\title{
5-aza-2'-deoxycytidine promotes migration of acute monocytic leukemia cells via activation of CCL2-CCR2-ERK signaling pathway
}

\author{
$\mathrm{XIA} \mathrm{XIAO}^{1,2^{*}}, \mathrm{QIAN} \mathrm{XU}^{1 *}, \mathrm{YAN} \mathrm{SUN}^{3}, \mathrm{ZIWEI} \mathrm{LU}^{4}, \mathrm{RUI} \mathrm{LI}^{5}, \mathrm{XINXIN} \mathrm{WANG}^{1}$, \\ XIN JIANG $^{1}$, GUIRONG ZHANG ${ }^{1}$ and YECHEN XIAO ${ }^{1}$
}

${ }^{1}$ Department of Biochemistry and Molecular Biology, College of Basic Medical Science, Jilin University,

Changchun, Jilin 130021; Departments of ${ }^{2}$ Ecsomatics and ${ }^{3}$ Oncology and Hematology,

The Second Hospital of Jilin University, Changchun, Jilin 130041; ${ }^{4}$ School of Clinical Medicine;

${ }^{5}$ Department of Radiology, School of Public Health, Jilin University, Changchun, Jilin 130021, P.R. China

Received April 28, 2016; Accepted March 31, 2017

DOI: $10.3892 / \mathrm{mmr} .2017 .6737$

\begin{abstract}
Aza) has been approved for clinical use in the treatment of myelodysplastic syndrome and acute myeloid leukemia (AML). It inhibits cell proliferation and induces cell differentiation by demethylating various genes, including tumor suppressor genes, transcription factors, and genes encoding cell cycle inhibitors. Although it has demonstrated efficacy in the clinic, drug resistance following 5-Aza treatment occurs. Cell migration and invasion following 5-Aza treatment are considered to be the key factors underlying drug resistance; however, there is currently limited information regarding the detailed mechanisms involved. In the present study, the THP-1 monocytic leukemia cell line was employed. The anti-leukemic functions of 5-Aza in THP-1 cells were first investigated. The results demonstrated that 5-Aza induced differentiation and inhibited THP-1 cell growth. Notably, 5-Aza significantly promoted THP-1 cell migration. Using reverse transcription-polymerase chain reaction, Western blot and enzyme-linked immunosorbent assay analyses, 5-Aza treatment was observed to upregulate the expression of chemokine (C-C motif) ligand 2 (CCL2) and $\mathrm{C}-\mathrm{C}$ chemokine receptor type 2 (CCR2) in THP-1 cells. In addition, the results demonstrated that CCL2 induced extracellular signal-regulated kinase (ERK) phosphorylation by CCR2 in 5-Aza-treated THP-1 cells. Treatment with a CCR2 or ERK inhibitor inhibited the 5-Aza-induced increase in THP-1
\end{abstract}

Correspondence to: Dr Yechen Xiao, Department of Biochemistry and Molecular Biology, College of Basic Medical Science, Jilin University, 126 Xinmin Street, Changchun, Jilin 130021, P.R. China E-mail: yechenxiao2400@126.com

*Contributed equally

Key words: 5-aza-2'-deoxycytidine, migration, differentiation, growth inhibition, acute monocytic leukemia cells cell migration. In conclusion, the results of the present study provide an insight into the molecular mechanism underlying the 5-Aza-induced increase in THP-1 cell migration, as well as a potential strategy to overcome drug resistance in AML therapy.

\section{Introduction}

Acute myeloid leukemia (AML) is a heterogeneous disease characterized by the malignant clonal proliferation of immature myeloid cells, which is associated with a poor overall prognosis (1). To date, only the acute promyelocytic leukemia subtype of AML has demonstrated improved therapeutic effects through the use of differentiation-inducing agents, such as all-trans retinoic acid and/or arsenic trioxide (2). However, there are currently no effective treatments for additional types of AML. Common chemotherapy drug regimens, such as anthracyclines combined with cytarabine, are not effective for the treatment of all AML patients, and eventually lead to the development of drug resistance (3).

In recent years, the demethylation agent, 5-aza-2'-deoxycytidine (5-Aza) has been approved by US Food and Drug Administration, and applied to treat myelodysplastic syndrome (MDS) and AML (4). 5-Aza induces the loss of methylation and reactivates silenced genes via the irreversible inhibition of DNA methyltransferases (5). A number of previous studies have reported that 5-Aza induces cell growth inhibition, differentiation and apoptosis of leukemia cells via distinct mechanisms (6-8). In particular, Ding et al (8) demonstrated that 5-Aza increases the sensitivity of multidrug-resistant HL-60/ADR leukemia cells to aclacinomycin via the epigenetic modulation of demethylation, which suggests 5-Aza appears to be safe and effective for the treatment of patients with high-risk AML. Although 5-Aza monotherapy demonstrates a high response rate in patients with AML, the complete remission rate remains low, and eventually all patients experience disease relapse $(9,10)$. Enhanced tumor invasiveness is considered to be an important reason underlying the clinical failure and disease progression that occurs following 5-Aza 
treatment $(10,11)$. However, the detailed mechanisms of 5-Aza-induced increases in tumor cell migration and invasion have not yet been completely elucidated.

In the present study, the effect of 5-Aza on the growth and differentiation of THP-1 monocytic leukemia cells was investigated. Of particular note, the molecular mechanism of 5-Aza-induced increases in THP-1 cell migration was proposed. The results demonstrated the opposing roles of 5-Aza in the treatment of acute monocytic leukemia, and present a potential strategy to inhibit the cell migration pathway in the clinical application of 5-Aza.

\section{Materials and methods}

Reagents. 5-Aza (catalog no. A3656) and the RS102895 (RS) C-C chemokine receptor type 2 (CCR2) inhibitor (catalog no. R1903) were purchased from Sigma-Aldrich; Merck Millipore (Darmstadt, Germany). Anti-CCR2 (catalog no. 12199S), anti-extracellular signal-regulated kinase (ERK) (catalog no. 4695S), anti-phosphorylated (p)-ERK (catalog no. 4376S), anti-AKT (catalog no. 9272S), anti-p-AKT (catalog no. 4058S), anti- $\beta$-actin (catalog no. 4967S) and horseradish peroxidase-conjugated secondary antibody (catalog no. 7074S) were purchased from Cell Signaling Technology, Inc. (Danvers, MA, USA). The phosphatidylinositol-4,5-bisphosphate 3-kinase (PI3K) inhibitor, LY294002 (catalog no. S1105), the c-Jun N-terminal kinase (JNK) inhibitor, SP600125 (catalog no. S1460), the p38 inhibitor, SB203580 (catalog no. S1076), the mitogen-activated protein kinase (MAPK)/ERK inhibitor, PD98059 (catalog no. S1177) and the NF- $\mathrm{B}$ inhibitor, BMS345541 (catalog no. 8044), were purchased from Selleck Chemicals (Houston, TX, USA). Transwell chambers were purchased from Corning Incorporated (Corning, NY, USA). The chemokine (C-C motif) ligand 2 (CCL2) enzyme-linked immunosorbent assay (ELISA) kit (catalog no. DCP00) was obtained from R\&D Systems, Inc. (Minneapolis, MN, USA). The bromodeoxyuridine (BrdU) kit (catalog no. 552598) was purchased from BD Biosciences (Franklin Lakes, NJ, USA). The phycoerythrin (PE)-conjugated anti-human cluster of differentiation 14 (CD14) antibody was purchased from eBioscience, Inc. (San Diego, CA, USA). THP-1 cell line was purchased from the American Type Culture Collection (Manassas, VA, USA). The bicinchoninic acid protein assay kit (catalog no. 23225) was purchased from Thermo Fisher Scientific, Inc., Waltham, MA, USA.

Migration assay. THP-1 cells were cultured in RPMI 1640 (Hyclone; GE Healthcare Life Sciences, Logan, UT, USA) supplemented with $10 \%$ fetal bovine serum (FBS; Biological industries USA, Inc., CT, USA), $100 \mathrm{U} / \mathrm{ml}$ ampicillin (Beijing Solarbio Science and Technology Co., Ltd., Beijing, China) and $100 \mathrm{U} / \mathrm{ml}$ streptomycin (Beijing Solarbio Science and Technology Co., Ltd.) at $37^{\circ} \mathrm{C}$ and $5 \% \mathrm{CO}_{2}$. Prior to stimulation, THP-1 cells were divided into different groups and treated with a certain drug according to experimental needs. In general, $1 \times 10^{6}$ pretreated cells were resuspended in $100 \mu 1$ serum-free RPMI 1640 media and transferred to the upper Transwell chamber of the 24-well plates in the absence or presence of a specific drug. Complete media (600 $\mu \mathrm{l})$ containing $20 \%$ FBS was added to the lower chamber. The plates were placed in the incubator for $48 \mathrm{~h}$ at $37^{\circ} \mathrm{C}$. The number of cells that had migrated to the lower chamber was then determined. An equal volume of $0.1 \%$ dimethyl sulfoxide (DMSO) that was used to reconstitute the drug was used as a negative control for all experiments.

Reverse transcription-polymerase chain reaction (RT-PCR) analysis. A total of $1 \times 10^{6}$ THP-1 cells were treated with $0.5 \mu \mathrm{M} 5$-Aza or DMSO for $48 \mathrm{~h}$. Total RNA was isolated from 5-Aza or DMSO treated THP-1 cells using Trizol Reagent (Thermo Fisher Scientific, Inc.). First-strand cDNA was synthesized using $2 \mu \mathrm{g}$ of total RNA in a $20 \mu \mathrm{l}$ reverse transcriptase reaction mixture using a cDNA synthesis kit (Beijing Transgen Biotech Co., Ltd., Beijing, China). The primers used for PCR were as follows: forward, 5'CTCATA GCAGCCACCTTCAT3' and reverse, 5'TCACAGCTTCTT TGGGACAC3' for CCL2; forward, 5'TCTCGTTCTCGGTTT ATCAG3' and reverse, 5'ATTCCCAAAGACCCACTCAT3' for CCR2; forward, 5'TGCACCACCAACTGCTTAGC3' and reverse, 5'GGCATGGACTGTGGTCATGA3' for GAPDH. Cycling conditions were as follows: An initial predenaturation step at $94^{\circ} \mathrm{C}$ for $5 \mathrm{~min}$, followed by 28 cycles of denaturation at $94^{\circ} \mathrm{C}$ for $30 \mathrm{sec}$, annealing at $57^{\circ} \mathrm{C}$ for $30 \mathrm{sec}$, extension at $72^{\circ} \mathrm{C}$ for $30 \mathrm{sec}$ and a final extension step at $72^{\circ} \mathrm{C}$ for $5 \mathrm{~min}$. The PCR product was confirmed by electrophoresis on $2 \%$ agarose gels, stained with $0.5 \mu \mathrm{g} / \mathrm{ml}$ ethidium bromide solution and detected using a Tanon 1600 ultraviolet detector.

Western blot analysis. A total of $2 \times 10^{6}$ THP-1 cells were treated with $0.5 \mu \mathrm{M} 5$-Aza or $0.1 \%$ DMSO for different time points at $4^{\circ} \mathrm{C}$. Total cellular protein $(100 \mu \mathrm{g})$ was extracted with radioimmunoprecipitation buffer (Thermo Fisher Scientific, Inc.) at $4^{\circ} \mathrm{C}$ and quantified using a bicinchoninic acid assay kit (Thermo Fisher Scientific, Inc.). Proteins were separated on $12 \%$ SDS-PAGE gels and transferred onto polyvinylidene difluoride membranes. The nonspecific binding of transferred proteins was blocked with PBS buffer containing 5\% non-fat milk powder for $2 \mathrm{~h}$ at room temperature. The membrane was probed with a 1:1,000 dilution of primary antibodies against p-ERK, ERK, p-AKT, AKT, CCR2 or $\beta$-actin overnight at $4^{\circ} \mathrm{C}$, prior to washing three times with Tris-buffered saline and $0.05 \%$ Tween 20 buffer. Membranes were subsequently probed with a 1:1,000 dilution of horseradish peroxidase-conjugated secondary antibody. Blotted proteins were detected using an enhanced chemiluminescence kit (Thermo Fisher Scientific, Inc.) and bands were quantified using Tanon Image software version 1.10 (Tanon Science and Technology Co., Ltd., Shanghai, China).

ELISA. A total of $1 \times 10^{6}$ THP-1 cells were treated with $0.5 \mu \mathrm{M}$ 5 -Aza for $96 \mathrm{~h}$. The culture medium was then collected and the concentration of CCL2 was determined by measuring the absorbance on a plate reader at a wavelength of $450 \mathrm{~nm}$, according to the manufacturer's protocol (R\&D Systems, Inc.).

Cell differentiation. A total of $2 \times 10^{5}$ THP-1 cells were cultured in Dulbecco's modified Eagle's medium containing 10\% FBS at $37^{\circ} \mathrm{C}$ in the presence of $0.1 \%$ DMSO or 0.1 or $0.5 \mu \mathrm{M} 5$-Aza for 6 days. During the incubation period, the cell culture media containing DMSO or 5-Aza were refreshed daily. Cells were 


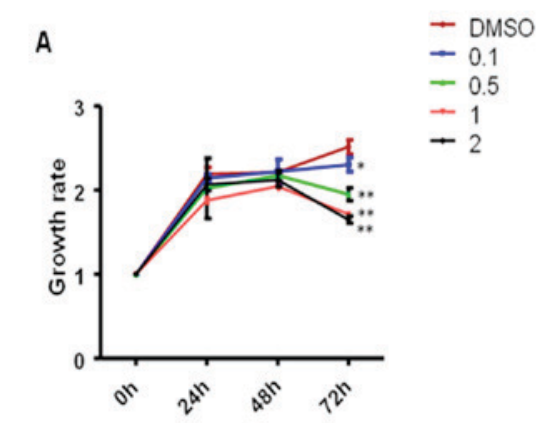

B

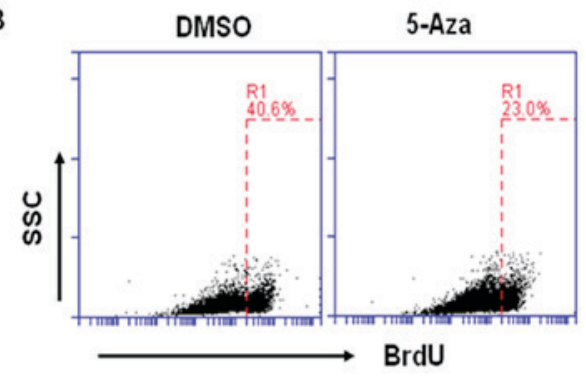

C

$\begin{array}{ll}C & \\ & \text { DMSO }\end{array}$

$0.1 \quad 0.5$

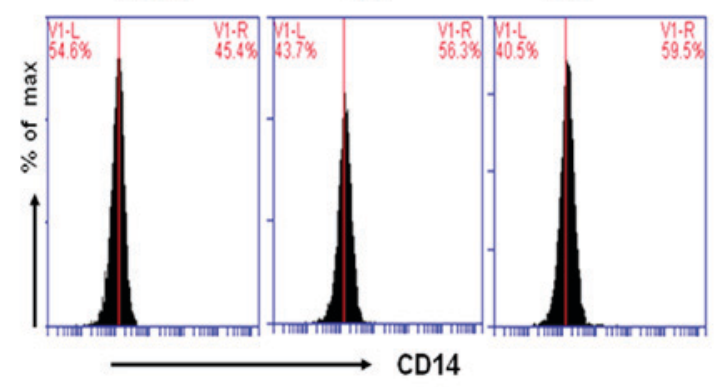

D

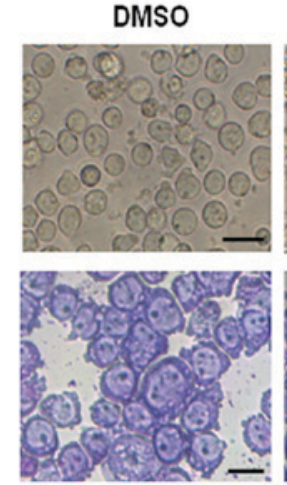

5-Aza

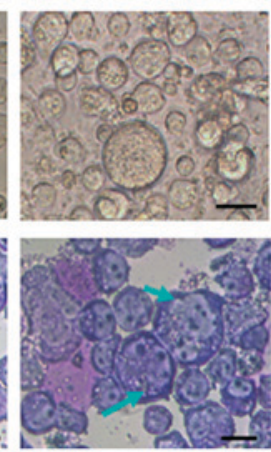

Figure 1. 5-Aza treatment induced growth inhibition and differentiation of THP-1 cells. (A) THP-1 cells were treated with DMSO or 0.5 $\mu$ M 5-Aza for 24 , 48 and $72 \mathrm{~h}$ and cell growth was assayed by using a cell counting kit-8 assay. (B) BrdU staining was performed on 5-Aza- and DMSO-treated THP-1 cells. (C) The expression level of CD14 in 5-Aza-treated THP-1 cells was assayed by flow cytometry. (D) Cell differentiation was visualized under a light microscope (upper panels) and following Wright-Giemsa staining (lower panel). The green arrows indicate differentiated cells with large volumes compared with the DMSO-treated cells. Scale bar $=10 \mu \mathrm{m}$. The results are presented as the mean \pm standard error of the mean $(\mathrm{n}=4-6)$, and pictures are representative of three independent experiments. "P<0.01 and ${ }^{* *} \mathrm{P}<0.001$ vs. DMSO group. 5-Aza, 5-aza-2'-deoxycytidine; DMSO, dimethyl sulfoxide; BrdU, bromodeoxyuridine; CD14, cluster of differentiation 14; SSC, side scatter.

stained with a 1:100 dilution of PE-conjugated anti-CD14 antibody for $20 \mathrm{~min}$ at room temperature, and were subsequently analyzed by flow cytometry using a BD Accuri C6 instrument (BD Biosciences). Cell morphology was observed by Wright-Giemsa staining. Briefly, $2 \times 10^{4}$ treated THP-1 cells were centrifuged at $300 \mathrm{x}$ g for $10 \mathrm{~min}$, piptted onto onto glass slides and then stained with Wright-Giemsa solution (catalog no. BA4017; Zhuhai Beisuo Biological Technology Co., Ltd., Zhuhai, China) for $15 \mathrm{~min}$ at room temperature. Cells were visualized with a light microscope (model BA1303IF) and captured using imaging software version 3.5 (Chongqing Lin Pei Photoelectric Instrument Co., Ltd., Chongqing, China). Images were captured from three independent experiments.

Cell counting kit-8 (CCK-8) assay. A total of $1 \times 10^{4} \mathrm{THP}-1$ cells were seeded in 96-well plates and treated with $0.1,0.5$, 1.0, $2.0 \mu \mathrm{M}$ 5-Aza or 0.1\% DMSO. Cell growth was assessed at $0,24,48$ and $72 \mathrm{~h}$ by adding $10 \mu \mathrm{l}$ CCK- 8 solution (BestBio, Shanghai, China) in the cell medium and incubated at $37^{\circ} \mathrm{C}$ for $2.5 \mathrm{~h}$, and the absorbance was measured using a plate reader at a wavelength of $450 \mathrm{~nm}$.

BrdU staining. A total of $1 \times 10^{6}$ THP-1 cells were treated with $0.5 \mu \mathrm{M} 5$-Aza or DMSO for $48 \mathrm{~h}$. DMSO was used as a negative control. BrdU was then directly added to the cell culture medium to final concentration of $10 \mu \mathrm{M}$. Cells was gently mixed and returned to the incubator for $30 \mathrm{~min}$ at $37^{\circ} \mathrm{C}$. The cells were subsequently collected, fixed and permeabilized, according to the BrdU kit protocol (BD Biosciences). Finally, cells were stained with a 1:50 dilution of allophycocyanin-conjugated BrdU antibody for $20 \mathrm{~min}$ at room temperature in the dark, and were analyzed by flow cytometry using a BD Accuri C6 instrument (BD Biosciences). Inhibition rate (IR) was calculated as follows: $\mathrm{IR}=\left(X^{-} Y\right) / X$, where $X$ and $Y$ are the percentage of $\mathrm{BrdU}^{+}$cells in the presence of the DMSO control and 5-Aza, respectively.

Statistical analysis. Data are presented as the mean \pm standard error of the mean, and were analyzed using GraphPad Prism 5.01 software (GraphPad Software, Inc., La Jolla, CA, USA). Comparison of the mean between 2 groups was achieved using the Student's t-test, and comparisons among multiple groups was achieved by one-way analysis of variance with a Dunnett's post hoc test. $\mathrm{P}<0.05$ was considered to indicate a statistically significant difference.

\section{Results}

5-Aza treatment induced differentiation and growth inhibition of THP-1 cells. A previous study demonstrated that 5-Aza induces differentiation and growth inhibition of HL60 human promyelocytic leukemia cells (12). THP-1 cells, which are driven by the mixed-lineage leukemia (MLL)-ALL1-fused gene from chromosome 9 (AF9) fusion gene, are derived from the M5 subtype of AML, and are different to HL60 cells in a number of respects, including the regulation of gene expression, signaling pathway involvement and pathogenesis. To investigate whether 5-Aza inhibits the proliferation of THP-1 
A

THP-1 cells pretreated with 5-Aza for $48 \mathrm{~h}$

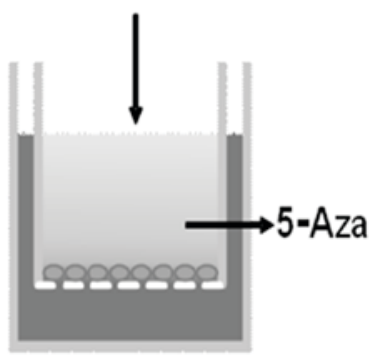

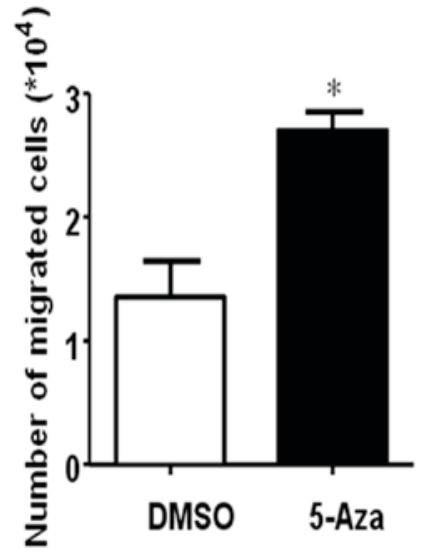

Figure 2.5-Aza treatment promotes THP-1 cell migration. (A) Schematic representation of the Transwell migration assay. (B) Using this experimental design, THP-1 cells were first pretreated with DMSO or $0.5 \mu \mathrm{M}$ 5-Aza for $48 \mathrm{~h}$, and the number of cells that had traversed the membrane was determined following $24 \mathrm{~h}$. Data are presented as the mean \pm standard error of the mean $(\mathrm{n}=4)$. ${ }^{*} \mathrm{P}<0.05$ vs. DMSO. 5-Aza, 5-aza-2'-deoxycytidine; DMSO, dimethyl sulfoxide.

A

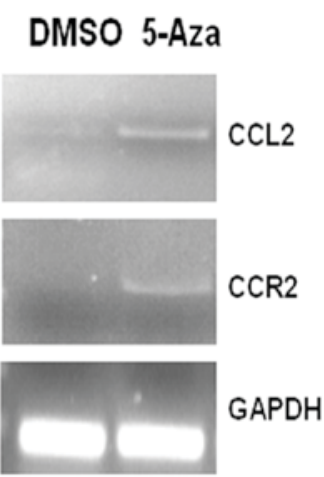

B

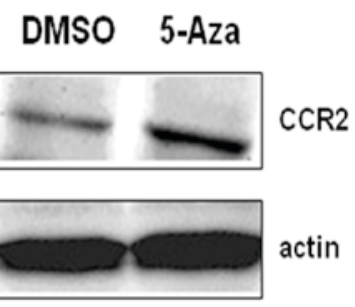

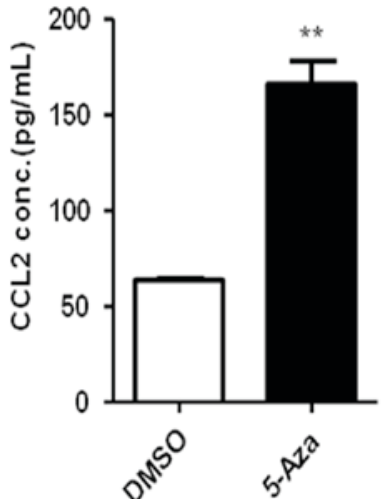

Figure 3. 5-Aza treatment increased the expression of CCL2 and CCR2. (A) THP-1 cells were treated with DMSO or 0.5 $\mu$ M 5-Aza for 72 h, and CCR2 and CCL2 expression levels were analyzed by reverse transcription-polymerase chain reaction. (B) Western blot analysis of CCR2 expression. (C) Enzyme-linked immunosorbent assay analysis of CCL2 levels in the culture medium. Data are presented as the mean \pm standard error of the mean ( $\mathrm{n}=4)$. ${ }^{* *} \mathrm{P}<0.01 \mathrm{vs}$. DMSO. 5-Aza, 5-aza-2'-deoxycytidine; CCL2, C chemokine (C-C motif) ligand 2; CCR2, C-C chemokine receptor type 2; DMSO, dimethyl sulfoxide.

cells, cell growth was evaluated by CCK-8 staining. As shown in Fig. 1A, 0.1, 0.5, 1 and $2 \mu \mathrm{M}$ 5-Aza treatment significantly inhibited THP-1 cell growth at $72 \mathrm{~h}$ when compared with the DMSO-treated control cells. However, at 24 and $48 \mathrm{~h}$, cell growth was not significantly altered among the 5-Aza-treated groups, which suggested that 5-Aza-induced cell growth inhibition may be dose- and time-dependent. Similarly, the results from BrdU staining demonstrated that 5-Aza significantly inhibited THP-1 cell proliferation, and the rate of growth inhibition reached $43 \%$ following treatment with $0.5 \mu \mathrm{M} 5$-Aza for 72 h (Fig. 1B).

In order to determine whether 5-Aza treatment induced differentiation of THP-1 cells, cells that had been pretreated with $0.5 \mu \mathrm{M} 5$-Aza for 6 days were stained with a PE-conjugated anti-CD14 antibody, and analyzed by flow cytometric analysis. The results demonstrated that the level of CD14 expression significantly increased in 0.1 or $0.5 \mu \mathrm{M}$ 5-Aza-treated cells when compared with that of DMSO-treated cells (Fig. 1C). However, when comparing the 0.5 and $0.1 \mu \mathrm{M}$ 5-Aza-treatment groups, they demonstrated similar results, suggesting that low concentrations of 5-Aza may be enough

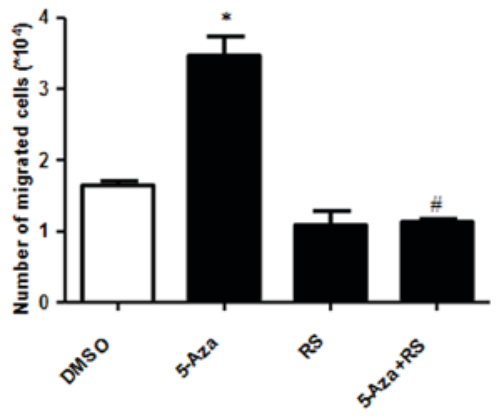

Figure 4. Inhibition of CCL2-CCR2 signaling reverses the 5-Aza-induced increase in THP-1 cell migration. THP-1 cells were treated with $0.5 \mu \mathrm{M}$ 5-Aza for $72 \mathrm{~h}$, and a cell migration assay was performed in the absence or presence of $10 \mu \mathrm{M}$ RS. Data are presented as the mean \pm standard error of the mean $(\mathrm{n}=4) .{ }^{*} \mathrm{P}<0.001$ vs. DMSO and ${ }^{\#} \mathrm{P}<0.001$ vs. 5 -Aza. CCL2, chemokine (C-C motif) ligand 2; CCR2, C-C chemokine receptor type 2; 5-Aza, 5-aza-2'-deoxycytidine; RS, RS102895; DMSO, dimethyl sulfoxide.

to induce cell differentiation. In addition, the morphology of 5-Aza-treated cells was observed under an inverted 


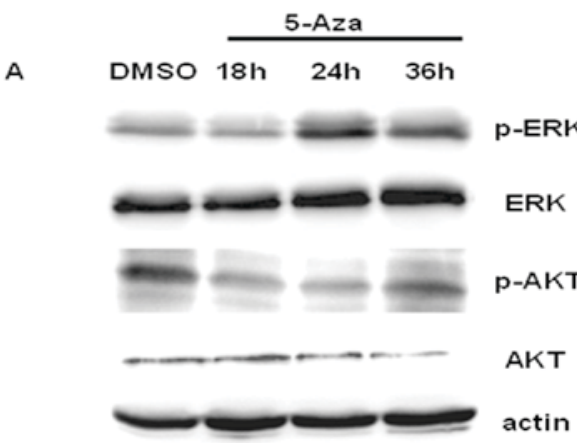

B
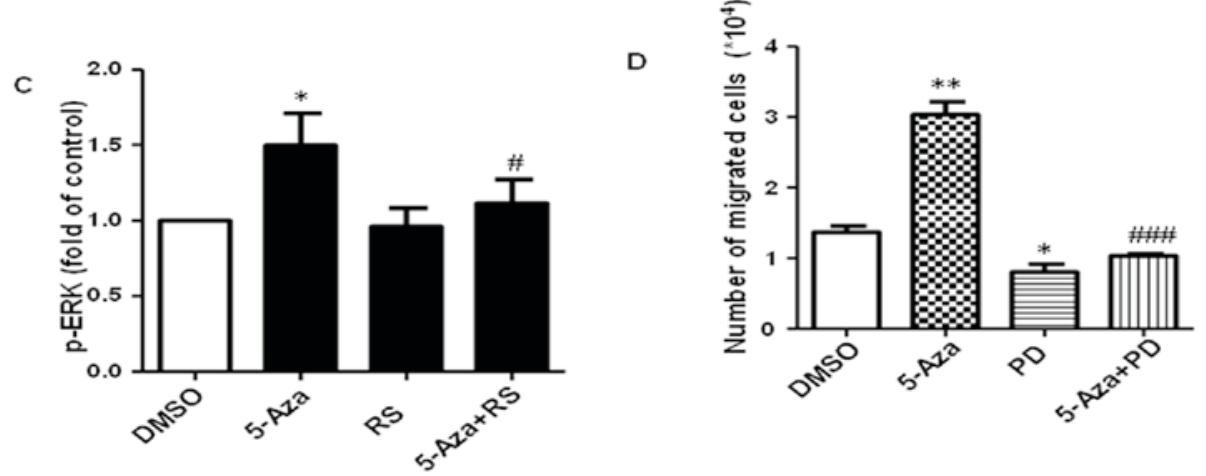

Figure 5. 5-Aza treatment promotes THP-1 cell migration by CCR2-mediated ERK activation. (A) THP-1 cells were treated with $0.5 \mu \mathrm{M} 5$-Aza for 18,24 or $36 \mathrm{~h}$ and the protein expression levels of p-ERK, ERK, p-AKT and AKT were analyzed by western blotting. (B) THP-1 cells were treated with $0.5 \mu \mathrm{M}$ 5-Aza with or without $10 \mu \mathrm{M}$ RS and activation of ERK was assayed by western blotting. (C) Quantification of p-ERK protein expression expressed relative to DMSO-treated controls. "P $<0.05$ vs. DMSO and ${ }^{*} \mathrm{P}<0.05$ vs. 5-Aza. (D) The migration ability of THP-1 cells following treatment with 5-Aza with or without $10 \mu \mathrm{M}$ PD. Data are presented as the mean \pm standard error of the mean $(\mathrm{n}=4) .{ }^{*} \mathrm{P}<0.05$ and ${ }^{* *} \mathrm{P}<0.01$ vs. DMSO and ${ }^{\# \# \#} \mathrm{P}<0.001$ vs. 5-Aza. 5-Aza, 5-aza-2'-deoxycytidine; CCR2, C-C chemokine receptor type 2; ERK, extracellular signal-regulated kinase; p-ERK, phosphorylated ERK; DMSO, dimethyl sulfoxide; RS, RS102895; PD, PD98059.

microscope and following Wright-Giemsa staining. As shown in Fig. 1D, a large proportion of 5-Aza-treated cells exhibited an increase in cell volume (indicated by green arrows) when compared with the DMSO-treated control group. These differentiated cells accounted for $\sim 20 \%$ of all 5-Aza-treated cells. These results suggest that 5-Aza treatment weakly induced the differentiation of THP-1 cells.

5-Aza promotes THP-1 cell migration. 5-Aza may promote cancer cell invasion by upregulating genes associated with this process. Bernal et al (10) reported that 5-Aza upregulated the expression of matrix metalloproteinase (MMP)-9 in MDS-derived AML cell lines, which may enhance invasiveness in vitro. In human fibrosarcoma cells, 5-Aza was observed to enhance tumor cell invasion via transcription-dependent modulation of MMP-1 expression (11). Therefore, the authors of the present study hypothesized that 5-Aza may promote THP-1 cell migration or invasion. In order to examine the effects of 5-Aza on THP-1 cell migration, THP-1 cells were treated with $0.5 \mu \mathrm{M} 5$-Aza for $48 \mathrm{~h}$, and then a migration assay was performed using Transwell chambers (Fig. 2A). The results demonstrated that 5-Aza significantly promoted THP-1 cell migration, and the number of migrated cells increased $\sim 2$-fold when compared with that of the DMSO control (Fig. 2B).

5-Aza-induced THP-1 cell migration was mediated by CCL2-CCR2 signaling. In order to identify the mechanism underlying the 5-Aza-induced increase in THP-1 cell migration, gene expression profiling of 5-Aza-treated THP-1 cells was performed, using a Phalanx OneArray ${ }^{\circledR}$ (Phalanx Biotech Group, San Diego, CA, USA).

The preliminary results demonstrated that the expression levels of numerous genes involved cell migration were altered, particularly CCR2 and CCL2 (data not shown). CCL2, also known as monocyte chemoattractant protein-1 (MCP-1), is an important chemotaxis molecule in monocytic cells that regulates monocyte migration via CCR2-mediated signaling pathways (13). To investigate whether the CCL2-CCR2 signaling pathway participates in the 5-Aza-induced increase in THP-1 cell migration, CCR2 and/or CCL2 expression was assayed by reverse transcription-polymerase chain reaction, western blotting and ELISA in THP-1 cells treated with $0.5 \mu \mathrm{M} 5$-Aza for $72 \mathrm{~h}$. The results demonstrated that CCL2 and CCR 2 mRNA levels were markedly upregulated in THP-1 cells following $72 \mathrm{~h}$ of 5-Aza treatment when compared with DMSO-treated controls (Fig. 3A). Similarly, an increase in CCR2 protein expression levels were observed in 5-Aza-treated THP-1 cells when compared with DMSO-treated controls (Fig. 3B). The ELISA results indicated that the concentration of CCL2 in cell culture medium increased 2.6-fold in the presence of 5-Aza when compared with that of control group (Fig. 3C). These results suggested that 5-Aza promoted the expression of CCL2 and CCR2 in THP-1 cells. To determine whether this increase in CCL2 and CCR2 expression was associated with 5-Aza-induced THP-1 cell migration, the migration capability of cells induced by 5-Aza in the presence of the RS CCR2 inhibitor was examined. As shown in Fig. 4, RS inhibited the 5-Aza-induced increase in THP-1 cell 
migration when compared with DMSO or RS-only treated groups. These results suggest that the 5-Aza-induced increase in cell migration may be dependent on the CCR2 signaling pathway.

5-Aza promotes THP-1 cell migration by CCR2-mediated ERK activation. Our studies demonstrated that the CCL2-CCR2 signaling pathway mediates 5-Aza-induced increases in THP-1 cell migration (Figs. 3 and 4). In order to investigate the mechanism underlying CCL2-CCR2-mediated regulation of this process, the role of CCL2-CCR2 downstream effectors was examined using the following specific inhibitors: The PI3K inhibitor, LY294002; the JNK inhibitor, SP600125; the p38 inhibitor, SB203580; the MAPK/ERK inhibitor PD; and the NF-кB inhibitor, BMS345541. Out of all inhibitors examine, only PD inhibited the 5-Aza-induced increase in THP-1 cell migration (data not shown). In addition, the results demonstrated that 5 -Aza promoted the expression of p-ERK following 24 and $36 \mathrm{~h}$ of treatment, but not following $18 \mathrm{~h}$ (Fig. 5A). This suggested that 5-Aza may activate ERK indirectly. The protein expression levels of p-AKT were reduced following treatment with 5-Aza for 18,24 or $36 \mathrm{~h}$ when compared with DMSO-treated controls (Fig. 5A), suggesting that 5-Aza-induced growth inhibition may be dependent on the suppression of AKT signaling pathway To further investigate whether CCL2-CCR2 promotes cell migration via activation of ERK, the expression levels of p-ERK were detected by western blot analysis following the treatment of cells with 5-Aza and/or RS. As shown in Fig. 5B and C, RS inhibited 5-Aza-induced ERK phosphorylation in THP-1 cells, which suggested that ERK is a downstream effector of CCL2-CCR2, and ERK activation depends on CCL2-CCR2 signaling. Notably, treatment of cells with the ERK inhibitor completely inhibited the 5-Aza-induced increase in THP-1 cell migration (Fig. 5D), which was similar to that of the CCR2 inhibitor.

\section{Discussion}

Several studies have reported that 5-Aza exerts a number of effects in different leukemia cell lines, including inhibition of cell proliferation and induction of differentiation $(6,12)$. THP-1, a monocytic leukemia cell line driven by the MLL-AF9 fusion gene, was employed for the purposes of the present study. This THP-1 cell line is different from additional previously reported cell lines including the gene expression status, signaling pathway involvement and pathogenesis, as these cells are driven by the MLL-AF9 fusion gene. The effect of 5-Aza treatment in THP-1 cells was investigated. Using CCK-8 assay analysis and BrdU staining, the results demonstrated that 5-Aza treatment inhibited THP-1 cell growth in vitro. The mechanism underlying the anti-proliferative effects of 5-Aza is hypothesized to involve the demethylation of genes, including tumor suppresser genes, cell cycle inhibitors and microRNAs (miRNAs). For instance, demethylation of p16INK4a by 5-Aza treatment induced cell growth inhibition of adult T-cell leukemia/lymphoma cells (14), HL60 cells (15), and an MDS cell line (16). In addition, previous studies have demonstrated that specific miRNAs may be involved in 5-Aza-induced cell growth arrest, such as miR-125a (17), miR-193a (18).
Ufkin et al (17) indicated that 5-Aza-induced restoration of miR-125a expression led to decreased cell proliferation, cell cycle progression, and enhanced apoptosis in NB4 cells via targeting of the the ErbB signaling pathway. In addition, Gao and colleagues (18) demonstrated that 5-Aza-induced restoration of miR-193a expression in AML inhibited c-kit expression, followed by inhibition of cell growth (18). Nishi et al (19) reported that the regulatory region for let-7b expression was hypermethylated in an MLL fusion gene-driven leukemic cell line, and cell growth was inhibited once its expression was partially recovered by 5-Aza treatment (19). It is therefore possible that this mechanism may be responsible for the 5-Aza-induced growth arrest of MLL-AF9-driven THP-1 cells in the present study. However, the possibility that additional molecular mechanisms are involved in the 5-Aza-induced growth arrest of THP-1 cells cannot be excluded.

In the current study, the level of differentiation of 5-Aza-induced THP-1 cells was determined by microscopy and by assessing the expression levels of CD14. The results indicated that 5-Aza produces a similar biological effect on cell differentiation in THP-1 cells to different types of AML cell lines $(12,20)$. 5-Aza is hypothesized to induce differentiation by inhibiting DNA methylation and upregulating the expression of transcription factors associated with differentiation. Koschmieder et al (20) demonstrated that 5-Aza induced upregulation of CD14 by enhancing Sp1 transcriptional activity. In the present study, 5-Aza treatment enhanced CD14 expression in THP-1 cells, which was associated with an increase in the number of differentiated cells. Therefore, the authors hypothesized that Sp1 may be critical for this process in THP-1 cells. PU.1 is a member of the Ezb transformation-specific sequence family of transcription factors and is expressed in granulocytic, monocytic and B-lymphoid cells $(21,22)$. 5-Aza-induced differentiation of K562 cells is dependent on the PU.1 expression levels (22). Alberich-Jordà et al (23) demonstrated that 5-Aza treatment restored the CCAAT-enhancer-binding protein $(\mathrm{C} / \mathrm{EBP}) \alpha-\mathrm{C} / \mathrm{EBP} \gamma$ balance and promoted the differentiation of primary human AML samples, which was characterized by $\mathrm{C} / \mathrm{EBP} \alpha$ silencing and $\mathrm{C} / \mathrm{EBP} \gamma$ upregulation in vitro. Therefore the authors of the present study will focus on investigating the function of these key transcription factors in 5-Aza-induced THP-1 differentiation in future studies.

Besides cell growth arrest and increased differentiation of THP1 cells by 5 -Aza treatment, the results of the current study demonstrated that 5-Aza significantly promoted THP-1 migration, and exhibited a 2-fold increase in cell migration when compared with the controls. To elucidate the underlying molecular mechanisms, gene expression profiling was performed using $0.5 \mu \mathrm{M}$ 5-Aza-treated THP-1 cells. Notably, a number of genes involved in cell migration and invasion were upregulated, including MMP9, CCR2 and CCL2. A previous study observed upregulated MMP9 expression in a 5-Aza-treated AML cell line and in samples derived from patients with disease relapse (10). MMP9 serves a critical role in AML by increasing the invasive properties of malignant myeloblasts, thus promoting clinical failure of the drug and progression to a more aggressive disease (10). The focus of the present study was to investigate the functions of CCL2 and CCR2 in the 5-Aza-induced increase in THP-1 cell migration. CCR2 is an important marker of monocytic cells, and the binding of 
CCL2 to CCR2 results in the chemotaxis of monocytes $(24,25)$. Therefore, the authors proposed that 5-Aza-induced THP-1 cell migration may be dependent on the CCL2-CCR2 axis. The results indicated that 5-Aza promoted CCR2 expression at the transcriptional and translational levels, and CCL2 is highly expressed in 5-Aza-treated THP-1 cells and secreted into the culture medium. The authors initially considered the possibility that 5-Aza may alter CCL2 and CCR 2 expression via demethylating-dependent or independent mechanisms (26). The level of CCR2 expression is affected by stress, such as intermittent hypoxia (24). Therefore, the authors proposed that 5-Aza may influence the expression of CCL2 and CCR2 via a demethylation-independent mechanism.

In the current study, the role of several signaling pathways involving JNK, ERK, p38 and NF- $\mathrm{BB}$, in the 5-Aza-induced increase in THP-1 cell migration were investigated using specific inhibitors. Out of all inhibitors analyzed, only the ERK inhibitor was observed to prevent the 5-Aza-induced increase in THP-1 cell migration. In obstructive sleep apnea (OSA), intermittent hypoxia induces the activation of ERK1/2 and p38 MAPK signaling pathways in monocytic cells, and increases CCR2 expression and MCP-1-induced chemotaxis of monocytes (24). This indicates that the CCL2-CCR2 axis may promote the chemotaxis and adhesion of monocytes (24). Yang et al (25) demonstrated that upregulation of CCL2 and CCR2 in low-metastatic nasopharyngeal carcinoma cell lines may promote cell migration and invasion. In addition, dual overexpression of CCL2/CCR2 activated the ERK1/2 signaling pathway, which sequentially upregulated MMP2 and MMP9. Similarly, Choi et al (27) demonstrated that the MAPK signaling pathway regulates the invasion and migration of monocytic cells. These reports are consistent with the results of the current study. The results further demonstrated that 5-Aza treatment induced cell migration by activating the CCL2-CCR2-ERK signaling pathway.

In conclusion, the present study demonstrated the important role of 5-Aza in promoting THP-1 cell differentiation and growth arrest, which reflects its promising application for the treatment of patients with MLL-AF9-driven AML in the clinic. In addition, the results indicated that 5-Aza might promote THP-1 cell migration by activating the CCL2-CCR2-ERK signaling pathway. These results will be important for the identification of the molecular mechanisms underlying the 5-Aza-induced increase in cell migration, which may lead to the development of strategies that reduce the likelihood of disease relapse in AML patients treated with 5-Aza. For instance, inhibition of the ERK or CCR2 signaling pathways in combination with 5-Aza may be an alternative method for the clinical treatment of patients with AML.

\section{Acknowledgements}

The present study was supported by the National Natural Science Foundation of China (grant no. 81570149).

\section{References}

1. Conway O'Brien E, Prideaux S and Chevassut T: The epigenetic landscape of acute myeloid leukemia. Adv Hematol 2014: 103175, 2014.
2. Luesink M, Pennings JL, Wissink WM, Linssen PC, Muus P, Pfundt R, de Witte TJ, van der Reijden BA and Jansen JH: Chemokine induction by all-trans retinoic acid and arsenic trioxide in acute promyelocytic leukemia: Triggering the differentiation syndrome. Blood 114: 5512-5521, 2009.

3. Radujkovic A, Dietrich S, Bochtler T, Krämer A, Schöning T, Ho AD, Dreger P and Luft T: Azacitidine and low-dose cytarabine in palliative patients with acute myeloid leukemia and high bone marrow blast counts-a retrospective single-center experience. Eur J Haematol 93: 112-117, 2014.

4. Plimack ER, Kantarjian HM and Issa JP: Decitabine and its role in the treatment of hematopoietic malignancies. Leuk Lymphoma 48: 1472-1481, 2007.

5. Vispé S, Deroide A, Davoine E, Desjobert C, Lestienne F, Fournier L, Novosad N, Bréand S, Besse J, Busato F, et al: Consequences of combining siRNA-mediated DNA methyltransferase 1 depletion with 5-aza-2'-deoxycytidine in human leukemic KG1 cells. Oncotarget 6: 15265-15282, 2015.

6. Zhang F, Dai X and Wang Y: 5-Aza-2'-deoxycytidine induced growth inhibition of leukemia cells through modulating endogenous cholesterol biosynthesis. Mol Cell Proteomics 11: M111.016915, 2012.

7. Shin DY, Park YS, Yang K, Kim GY, Kim WJ, Han MH, Kang HS and Choi YH: Decitabine, a DNA methyltransferase inhibitor, induces apoptosis in human leukemia cells through intracellular reactive oxygen species generation. Int J Oncol 41: 910-918, 2012.

8. Ding B, Wang Z, Jiang X, Li X, Wang C, Zhong Q, Jiang L, Dai M, Zhang YU, Wei QI and Meng F: Palliative chemotherapy followed by methylation inhibitor in high-risk acute myeloid leukemia: An in vitro and clinical study. Mol Clin Oncol 3: 1139-1144, 2015.

9. Fenaux P, Mufti GJ, Hellstrom-Lindberg E, Santini V, Finelli C, Giagounidis A, Schoch R, Gattermann N, Sanz G, List A, et al: Efficacy of azacitidine compared with that of conventional care regimens in the treatment of higher-risk myelodysplastic syndromes: A randomised, open-label, phase III study. Lancet Oncol 10: 223-232, 2009.

10. Bernal T, Moncada-Pazos A, Soria-Valles C and Gutiérrez-Fernández A: Effects of azacitidine on matrix metalloproteinase-9 in acute myeloid leukemia and myelodysplasia. Exp Hematol 41: 172-179, 2013.

11. Poplineau M, Schnekenburger M, Dufer J, Kosciarz A, Brassart-Pasco S, Antonicelli F, Diederich $M$ and Trussardi-Régnier A: The DNA hypomethylating agent, 5-aza-2'-deoxycytidine, enhances tumor cell invasion through a transcription-dependent modulation of MMP-1 expression in human fibrosarcoma cells. Mol Carcinog 54: 24-34, 2015.

12. Hassan HT, Veit A and Maurer HR: Synergistic interactions between differentiation-inducing agents in inhibiting the proliferation of HL-60 human myeloid leukaemia cells in clonogenic micro assays. J Cancer Res Clin Oncol 117: 227-231, 1991.

13. Deshmane SL, Kremlev S, Amini S and Sawaya BE: Monocyte chemoattractant protein-1 (MCP-1): An overview. J Interferon Cytokine Res 29: 313-326, 2009.

14. Uenogawa K, Hatta Y, Arima N, Hayakawa S, Sawada U, Aizawa S, Yamamoto T and Takeuchi J: Azacitidine induces demethylation of p16INK4a and inhibits growth in adult T-cell leukemia/lymphoma. Int J Mol Med 28: 835-839, 2011.

15. Su Y, Xu H, Xu Y, Yu J, Xian Y and Luo Q: Azacytidine inhibits the proliferation of human promyelocytic leukemia cells (HL60) by demethylation of MGMT, DAPK and p16 genes. Hematology 17: 41-46, 2012.

16. Kimura S, Kuramoto K, Homan J, Naruoka H, Ego T, Nogawa M, Sugahara $\mathrm{S}$ and Naito H: Antiproliferative and antitumor effects of azacitidine against the human myelodysplastic syndrome cell line SKM-1. Anticancer Res 32: 795-798, 2012.

17. Ufkin ML, Peterson S, Yang X, Driscoll H, Duarte C and Sathyanarayana P: miR-125a regulates cell cycle, proliferation, and apoptosis by targeting the ErbB pathway in acute myeloid leukemia. Leuk Res 38: 402-410, 2014.

18. Gao XN, Lin J, Li YH, Gao L, Wang XR, Wang W, Kang HY, Yan GT, Wang LL and Yu L: MicroRNA-193a represses c-kit expression and functions as a methylation-silenced tumor suppressor in acute myeloid leukemia. Oncogene 30: 3416-3428, 2011.

19. Nishi M, Eguchi-Ishimae M, Wu Z, Gao W, Iwabuki H, Kawakami S, Tauchi H, Inukai T, Sugita K, Hamasaki Y, et al: Suppression of the let-7b microRNA pathway by DNA hypermethylation in infant acute lymphoblastic leukemia with MLL gene rearrangements. Leukemia 27: 389-397, 2013. 
20. Koschmieder S, Agrawal S, Radomska HS, Huettner CS Tenen DG, Ottmann OG, Berdel WE, Serve HL and Müller-Tidow C: Decitabine and vitamin D3 differentially affect hematopoietic transcription factors to induce monocytic differentiation. Int J Oncol 30: 349-355, 2007.

21. Chen HM, Zhang P, Voso MT, Hohaus S, Gonzalez DA, Glass CK, Zhang DE and Tenen DG: Neutrophils and monocytes express high levels of PU.1 (Spi-1) but not Spi-B. Blood 85: 2918-2928, 1995.

22. Aoyama S, Nakano H, Danbara M, Higashihara M, Harigae H and Takahashi S: The differentiating and apoptotic effects of 2-aza-5'-deoxycytidine are dependent on the PU.1 expression level in PU.1-transgenic K562 cells. Biochem Biophys Res Commun 420: 775-781, 2012

23. Alberich-Jordà M, Wouters B, Balastik M, Shapiro-Koss C, Zhang H, Di Ruscio A, Radomska HS, Ebralidze AK, Amabile G, Ye M, et al: $\mathrm{C} / \mathrm{EBP} \gamma$ deregulation results in differentiation arrest in acute myeloid leukemia. J Clin Invest 122: 4490-4504, 2012.

24. Chuang LP, Chen NH, Lin SW, Chang YL, Liao HR, Lin YS, Chao IJ, Lin Y and Pang JH: Increased C-C chemokine receptor 2 gene expression in monocytes of severe obstructive sleep apnea patients and under intermittent hypoxia. PLoS One 9: e113304, 2014.
25. Yang J, Lv X, Chen J, Xie C, Xia W, Jiang C, Zeng T, Ye Y, Ke L, $\mathrm{Yu}$ Y, et al: CCL2-CCR2 axis promotes metastasis of nasopharyngeal carcinoma by activating ERK1/2-MMP2/9 pathway. Oncotarget 7: 15632-15647, 2016.

26. Nishioka $C$, Ikezoe T, Yang J, Udaka $K$ and Yokoyama $A$ : Simultaneous inhibition of DNA methyltransferase and histone deacetylase induces p53-independent apoptosis via down-regulation of Mcl-1 in acute myelogenous leukemia cells. Leuk Res 35: 932-939, 2011.

27. Choi YJ, Yoon JH, Cha SW and Lee SG: Ginsenoside Rh1 inhibits the invasion and migration of THP-1 acute monocytic leukemia cells via inactivation of the MAPK signaling pathway. Fitoterapia 82: 911-919, 2011 